\section{Trends in the incidence and mortality of cancer in Saudi Arabia}

\author{
Mohammad A. Althubiti, MSc, PhD, \\ Mohamed M. Nour Eldein, MSc, PhD.
}

\section{ABSTRACT}

Objectives: To analyze the overall trends in cancer incidence and mortality in Saudi Arabia between 1990 and 2016.

Methods: Data were retrieved through a Global Burden of Disease (GBD, 2016) database (Viz Hub) that is developed by the Institute for Health Metrics and Evaluation at the University of Washington.

Results: The incidence of cancer increased around 26-fold for thyroid cancer; approximately 10-fold in breast, colon, bladder and uterine cancers; 8-fold for prostate cancer; 5-fold for renal cancer; 4-fold for pancreatic and ovarian cancer; 3.5-fold for lung cancer; 3-fold for liver cancer, and 2 folds for lymphoma, leukemia and gastric cancer. There was also an increase in the percentage of mortality due to cancer during this period. However, we noticed that the percentage of deaths due to cancer decreased after 70 years of age in Saudi Arabia population.

Conclusion: The increases in the incidence of different types of cancer in the past decade could be due to the revolutionary change in socioeconomic status that has occurred in Saudi Arabia; therefore, a national plan should be established for cancer prevention, screening and therapy. Concerning mortality, the decrease in its percentage among elderly people could be due to biological factors that should be investigated in the future.

Saudi Med J 2018; Vol. 39 (12): 1259-1262 doi: 10.15537/smj.2018.12.23348

T here has been a remarkable development in the socioeconomic status in Saudi Arabia in the past 40 years. This development has encouraged the government to establish a plan for improving the medical care of its citizens. During this societal growth and change, people's lifestyles have also been modified, including changes in the prominence of factors such as sedative lifestyles, processed food consumption, and other behaviours. ${ }^{1}$ These lifestyle modifications are believed to be responsible for increases in the incidence of many diseases, such as cancer. ${ }^{2}$ In Saudi Arabia, the age standard rate of cancer incidence in 2004 was 57.2 per $100000 .^{3}$ In a country such as Saudi Arabia, where more than half of the population is younger than 29 years old, ${ }^{4}$ more effort should be dedicated to revealing the incidence and mortality of cancer in society in order to implement a management, screening, and prevention strategy in the country. This would ultimately help in understanding cancer trends, which could help in efforts to manage the incidence and mortality of cancer in the future. For this purpose, we used Global Burden of Disease (GBD 2016) data ${ }^{5}$ to assess the trends in the incidence and mortality of different cancer types in Saudi Arabia between 1990 and 2016.

Methods. Data were retrieved through a GBD compare database (Viz Hub) that was available online and developed by the Institute for Health Metrics and Evaluation at the University of Washington. ${ }^{5}$ The database was created based on the previous GBD studies. ${ }^{5}$ Global Burden of Disease compare data were gathered from vital statistics including the World Health Organization mortality database, the United Nations demographic earbook, individual publications from national ministries of health, and other sources. We searched for the incidence and the mortality of cancer record of Saudi Arabia in the period between 1990 to 2016 in GBD compare database. We included all Saudis that were recorded in the database for mortality and incidence of cancer in this period. In retrospective, we searched for the incidence of 21 types of cancer in both genders in the following order: leukemia; multiple myeloma; lymphoma; thyroid cancer; brain and nervous system cancers; bladder cancer; kidney cancer; prostate cancer; ovarian cancer; uterine cancer; cervical cancer; breast cancer; tracheal, bronchus and lung cancer; pancreatic cancer; gallbladder and biliary tract cancers; liver cancer; colon and rectum cancers; stomach cancer; esophageal cancer; nasopharyngeal cancer; and lip and oral cavity cancers in the period between 1990 to 2016 . We also searched for the mortality rates due to these 21 cancer types in this time period. The percentage of deaths due to cancer was calculated, and the population was divided into 5 groups as follows: 0-14 years, 15-49 years, 50-69 years, 70-89 years and above 90 years.

Disclosure. Authors have no conflict of interests, and the work was not supported or funded by any drug company. 
Statistical analysis. The statistical analysis and figures were carried out using Prism 7 (GraphPad) for Mac version.

Results. The incidence of cancer has increased in recent years in Saudi Arabia. The trend in the incidence of different cancer types in each year from 1990 to 2016 in Saudi Arabia was analyzed. We looked at 21 types of cancer, namely, leukemia, multiple myeloma, lymphoma, thyroid cancer, brain cancer, bladder cancer, kidney cancer, prostate cancer, ovarian cancer, uterine cancer, cervical cancer, breast cancer, lung cancer, pancreatic cancer, gallbladder cancer, liver cancer, colorectal cancer, stomach cancer, oesophageal cancer, nasopharyngeal cancer, and lip and oral cavity cancers, as shown in Figure 1.

Thyroid cancer showed a dramatic increase in new cases, from 71 in 1990 to 1900 in 2016, a 26-fold increase. The incidence of breast cancer was 350 cases in 1990, and it increased to 3400 cases in 2016, a 10 -fold increase. Colorectal cancer also showed a high incidence rate between 1990 and 2016, increasing from 300 to 2400 cases, respectively and a 10 -fold increase in new cases. In addition, the incidence of bladder cancer was 120 in 1990 and increased 10-fold to 850 cases in 2016. Prostate cancer also showed a high incidence rate, increasing from 150 to 1300 cases between 1990 and 2016, an 8-fold increase in incidence. Kidney cancer increased from 120 to 650 cases, a 5-fold increase.
Pancreatic cancer showed an increase in incidence from 170 to 700 cases, a 4-fold increase. For lung cancer, there was a rise in incidence between 1990 and 2016 from 350 to 1200 cases, a 3.5-fold increase. For liver cancer, there was an increase in the incidence between these years from 500 to 1400 cases, an approximately 3 -fold increase. For lymphoma and nervous system cancers, there was an increase in incidence from approximately 300 to 1000 cases between 1990 and 2016, respectively, a 3-fold increase. Leukemia showed a moderate elevation in incidence from 500 to 1000 cases in 1990 and 2016, respectively, only a 2-fold increase. The remaining cancers showed different trends in the incidence in 1990 and 2016, such as stomach cancer, 260 to 550 cases; ovarian cancer, 100 to 400 cases; uterine cancer, 44 to 450 cases; lip and oral cavity cancers, 100 to 300 cases; and multiple myeloma, 40 to 270 cases, respectively, as shown in Figure 1.

The number of deaths due to cancer has increased in recent years in Saudi Arabia. The percentage of deaths due to cancer increased steadily between 1990 and 2016 . In 1990, the total percentage of deaths due to cancer was approximately $5 \%$, and the number increased and reached 12\% in 2016, as shown in Figure 2.

To study the percentages of death due to cancer in different age groups, the population was categorized into 7 groups: 0-14 years, 15-49 years, 50-69 years, 70-89 years and above 90 years, as shown in Figure 3. In the 0-14 years group, the percentage of death due to cancer
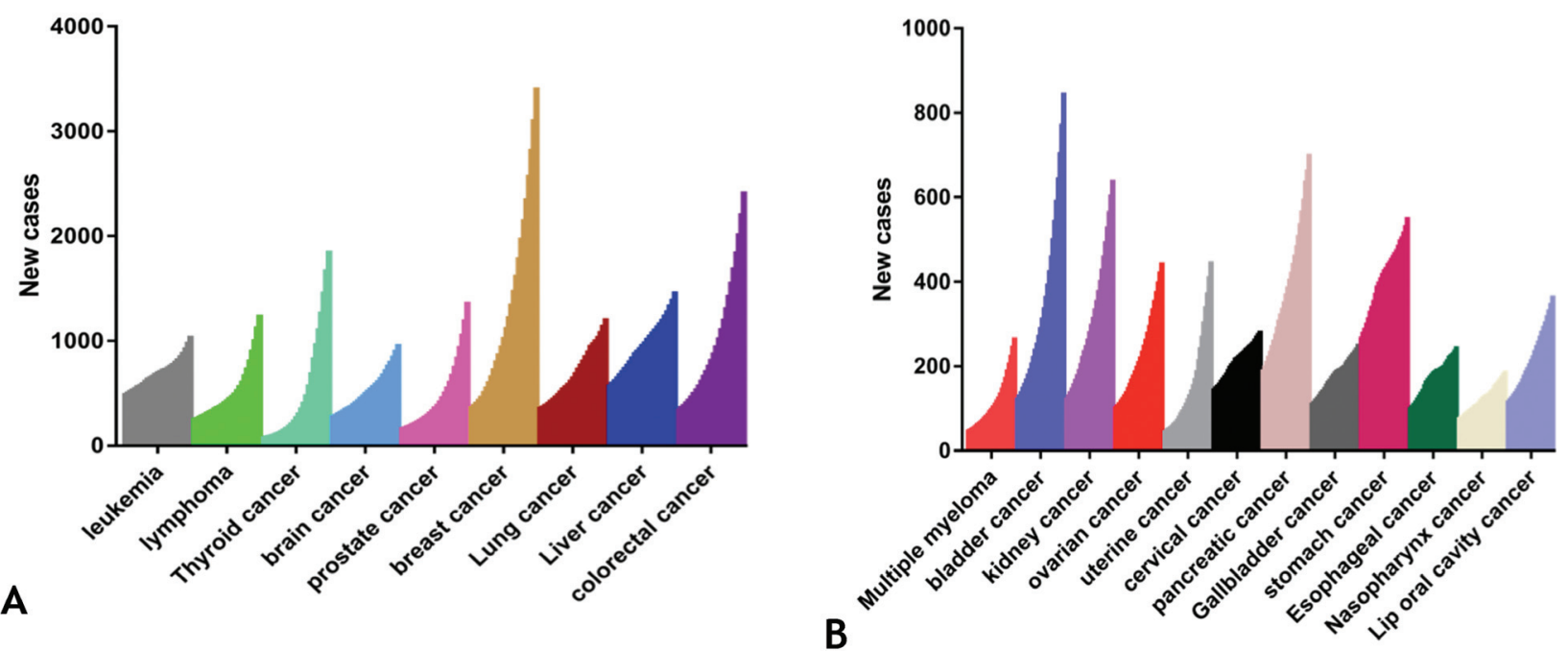

Figure 1 - The incidence of different cancer types that increased to A) $>1000$ new cases by 2016 and B) $<1000$ cases by 2016 . 


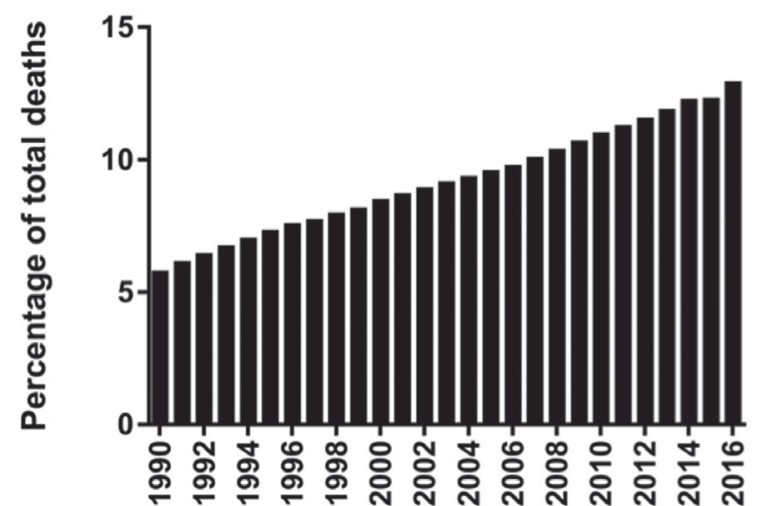

Figure 2 - The percentage of deaths due to cancer between 1990 and 2016.

was approximately 5\%. In the 15-49 years group, the percentage of death due to cancer was approximately $10 \%$. An increase was noticed in the percentage of death in the next group, 50-69 years, to $19 \%$. However, a reduction in the percentages of death in the following groups, those $70-89$ and above 90 years, to $14 \%$ and $70 \%$, respectively, was observed, as shown in Figure 3.

Discussion. There was a remarkable increase in the incidence and mortality of cancer in Saudi Arabia during the period between 1990 and 2016. The increase in the total population from 16 million to 30 million from 1990 to $2016{ }^{6}$ does not justify this increase. However, there are some probable reasons behind the rise in the number of deaths. Factors such as the consumption of calories have shown to be linked with cancer incidence. ${ }^{7}$ In Saudi Arabia, there was an increase in caloric intake from $2850 \mathrm{Kcal} /$ person/day in the period between 1990 and 1992 to $3120 \mathrm{Kcal} /$ person/day in the period between 2006 and 2008. ${ }^{1}$ This increase in caloric intake could have a role in the spreading of many diseases, such as cancer. ${ }^{7}$

Breast cancer showed a sharp increase during this period of time. Breast cancer screening has been shown to be a very effective approach to early diagnosis of breast cancer. ${ }^{8}$ In addition, screening of the high-risk population has a role in reducing breast cancer incidence. ${ }^{9}$ A study conducted in 2013 in Saudi Arabia investigated the awareness of breast cancer among women, including factors such as self-clinical exams and mammography. ${ }^{10}$ Roughly $89 \%$ of the participants had not had a clinical exam for breast cancer in the past year, and $92 \%$ had never undergone a mammogram. ${ }^{11}$ This

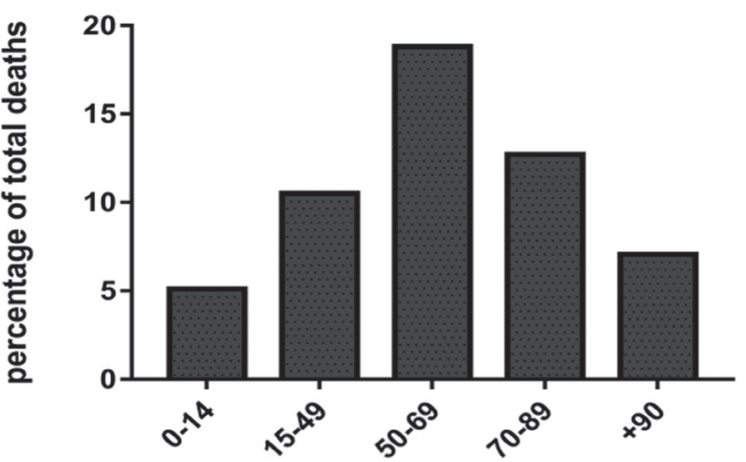

Figure 3 - The percentages of death due to cancer in different age groups: 0-14 years, 15-49 years, 50-69 years, 70-89 years and above 90 years.

underscores the increase in deaths due to breast cancer in Saudi Arabia, compared to, for example, that in the United States, where breast cancer screening awareness has been thoroughly established. ${ }^{12}$

The data also revealed a remarkable rise in the incidence of colorectal cancer in the past 26 years. The incidence of colorectal cancer varies globally. In Europe, its incidence is constant, while it is declining in the United States. ${ }^{13}$

The results showed that the incidence of thyroid cancer had the greatest increase between 1990 and 2016 in Saudi Arabia. The exact etiological factors of thyroid cancer have not been discovered. Worldwide, the incidence of thyroid cancer has increased without any differences in the mortality rate. ${ }^{14}$ Therefore, more investigations should be conducted to reveal the risk factors of thyroid cancer in Saudi Arabia.

Liver cancer and pancreatic cancer also showed increases in the incidence and mortality rate in the period between 1990 and 2016. The main risk factor of developing liver cancer is chronic infection with hepatitis B \& C. However, in Saudi Arabia, an attempt to control hepatitis infection resulted in the eradication of cases. ${ }^{15}$ The second main risk factors are obesity and insulin resistance; a study in 2007 showed that 33\% of the Saudi population was obese, ${ }^{16}$ and the number has increased since that time, which could explain the increase in liver cancer incidence. Concerning pancreatic cancer, longstanding diabetes is among the factors that play a role in its development. ${ }^{17}$ Prostate cancer also showed a very high incidence and mortality in Saudi Arabia. Despite the widespread use of prostate-specific antigen (PSA) as a screening test for its early detection, its incidence 
is still high. ${ }^{18}$ There are several studies that explain the usefulness and limitations of PSA that should be taken into account when screening for prostate cancer.

Data have shown that the percentage of people dying from cancer decreased after 70 years. After these ages, deaths in the Saudi population were due to other factors. There is no clear reason behind this reduction in the percentage of deaths at older ages; however, several reasons could play a role. For example, people who live to an old age could have good immune systems that eradicate the growth of cancer cells, in addition, this population may have a more effective DNA repair mechanism that prevents normal cell transformation. ${ }^{19}$ Therefore, more studies should be performed to study the biology of elderly people in Saudi Arabia and to compare them with middle-aged people in order to elucidate the possible preventive mechanisms among elderly people.

The limitations of this study are its retrospective nature and inability to determine if there are regional variations of the incidence and mortality due cancer in Saudi Arabia.

In conclusion, the results showed an increase in the incidence and mortality of cancer in the past 27 years. However, some cancer types were more prevalent than others. Therefore, these data can be exploited to study the actual trends of cancers in Saudi Arabia in order to implement a national strategy for cancer prevention, screening and therapy.

Acknowledgment. This work was supported by Biochemistry Department, Faculty of Medicine, Umm Al-Qura University, Makkah, Saudi Arabia.

\section{Received 11th July 2018. Accepted 26th September 2018.}

From the Department of Biochemistry (Althubiti, Eldein), Umm Al-Qura University, Makkah, Kingdom of Saudi Arabia, and from the Faculty of Medicine (Eldein), Ain Shams University, Cairo, Egypt.

Address correspondence and reprints request to: Dr. Mohammad Althubiti, Biochemistry Department, Faculty of Medicine, Umm Al-Qura University, Makkah, Kingdom of Saudi Arabia. E-mail: mathubiti@uqu.edu.sa

ORCID ID: orcid.org/0000-0002-9738-3188

\section{References}

1. Food Consumption: Food and Agriculture Organization of United Nation; 2011 [cited 2018 Feb 06]. Available from: http:// www.fao.org/fileadmin/.../FoodConsumptionNutrients_en.xls.

2. Anand P, Kunnumakkara AB, Sundaram C, Harikumar KB, Tharakan ST, Lai OS, et al. Cancer is a preventable disease that requires major lifestyle. Pharmaceutical Research 2008; 25: 2097-2116.
3. Ibrahim E, Bin SB, Banjar L, Awadalla S, Abomelha MS. Current and future cancer burden in Saudi Arabia: meeting the challenge. Hematol Oncol Stem Cell Ther 2008; 1: 210-215.

4. General Authority for Statistics. Demography Survey 2016. [cited 2016]. Available from: https://www.stats.gov.sa/sites/ default/files/en-demographic-research-2016_2.pdf.

5. Institiute for Health Metrics and Evaluation. GBD compare Viz Hub. University of Washington (WA) 2018. [updated 2018 Nov 08; cited 2018]. Available from: https://vizhub.healthdata. org/gbd-compare/.

6. United Nations. Desa Population Division. World Population Prospects 2017. [cited 2018]. Available from: https://esa. un.org/unpd/wpp/DataSources/.

7. Kushi LH, Byers T, Doyle C, Bandera EV, McCullough M, McTiernan A, et al. American Cancer Society guidelines on nutrition and physical activity for cancer prevention: reducing the risk of cancer with healthy food choices and physical activity. CA Cancer J Clin 2006; 56: 254-281.

8. Tabar L, Fagerberg CJ, Gad A, Baldetorp L, Holmberg LH, Grontoft $\mathrm{O}$, et al. Reduction in mortality from breast cancer after mass screening with mammography. Randomised trial from the Breast Cancer Screening Working Group of the Swedish National Board of Health and Welfare. Lancet 1985; 1: 829-832.

9. Harding C, Pompei F, Burmistrov D, Welch HG, Abebe R, Wilson R. Breast cancer screening, incidence, and mortality across US counties. JAMA Intern Med 2015; 175: 1483-1489.

10. El Bcheraoui C, Basulaiman M, Wilson S, Daoud F, Tuffaha M, AlMazroa MA, et al. Breast cancer screening in Saudi Arabia: free but almost no takers. PLoS One 2015; 10: e0119051.

11. Sengmany K, Singh J, Stewart GD, Conn PJ, Christopoulos A, Gregory KJ. Biased allosteric agonism and modulation of metabotropic glutamate receptor 5: Implications for optimizing preclinical neuroscience drug discovery. Neuropharmacology 2016; 115: 60-72.

12. National Cancer Institute. Surveillance, Epidemiology and Results Program. Cancer stat facts: female breast cancer. [cited 2018]. Available from: https://seer.cancer.gov/statfacts/html/ breast.html.

13. Jemal A, Thun MJ, Ries LA, Howe HL, Weir HK, Center $\mathrm{MM}$, et al. Annual report to the nation on the status of cancer, 1975-2005, featuring trends in lung cancer, tobacco use, and tobacco control. J Natl Cancer Inst 2008; 100: 1672-1694.

14. Rahman GA. Extent of surgery for differentiated thyroid cancer: recommended guideline. Oman Med J 2011; 26: 56-58.

15. Abdo AA, Sanai FM, Al-Faleh FZ. Epidemiology of viral hepatitis in Saudi Arabia: are we off the hook. Saudi J Gastroenterol 2012; 18: 349-357.

16. Al Othaimeen AI, Al-Nozha M, Osman AK. Obesity: an emerging problem in Saudi Arabia. Analysis of data from the National Nutrition Survey. East Mediterr Health J 2007; 13: 441-448.

17. Huxley R, Ansary-Moghaddam A, Berrington de Gonzalez A, Barzi F, Woodward M. Type-II diabetes and pancreatic cancer: a meta-analysis of 36 studies. Br J Cancer 2005; 92: 2076-2083.

18. Fitzpatrick JM. Management of localized prostate cancer in senior adults: the crucial role of comorbidity. BJU Int 2008; 101 Suppl 2: 16-22.

19. Hoeijmakers JH. Genome maintenance mechanisms for preventing cancer. Nature 2001; 411: 366-374. 